\title{
A WFC3 GRISM EMISSION LINE REDSHIFT CATALOG IN THE GOODS-SOUTH FIELD
}

\author{
Aaron M. Morris ${ }^{1}$, Dale D. Kocevski ${ }^{1}$, Jonathan R. Trump ${ }^{2,16}$, Benjamin J. Weiner ${ }^{3}$, Nimish P. Hathi ${ }^{4}$, \\ Guillermo Barro ${ }^{5}$, Tomas Dahlen ${ }^{6}$, Sandra M. Faber ${ }^{5}$, Steven L. Finkelstein ${ }^{7}$, Adriano Fontana ${ }^{8}$, \\ Henry C. Ferguson ${ }^{6}$, Norman A. Grogin ${ }^{6}$, Ruth Grützbauch ${ }^{9,10}$, Yicheng Guo ${ }^{5,11}$, Li-Ting Hsu ${ }^{12}$, \\ Anton M. Koekemoer ${ }^{6}$, David C. Koo ${ }^{5}$, Bahram Mobasher ${ }^{13}$, Janine Pforr ${ }^{14,15}$, Mara Salvato ${ }^{12}$, \\ TOMMY WiKLIND ${ }^{6}$, AND STIJN Wuyts ${ }^{12}$ \\ ${ }^{1}$ Department of Physics and Astronomy, University of Kentucky, Lexington, KY 40506, USA; aaron.morris2@uky.edu \\ ${ }^{2}$ Department of Astronomy and Astrophysics, Pennsylvania State University, University Park, PA 16802, USA \\ ${ }^{3}$ Steward Observatory, 933 N. Cherry Street, University of Arizona, Tucson, AZ 85721, USA \\ ${ }^{4}$ Aix Marseille Université, CNRS, LAM (Laboratoire d'Astrophysique de Marseille) UMR 7326, F-13388, Marseille, France \\ ${ }^{5}$ UCO/Lick Observatory and Department of Astronomy and Astrophysics, University of California, Santa Cruz, CA 95064, USA \\ ${ }^{6}$ Space Telescope Science Institute, Baltimore, MD 21218, USA \\ ${ }^{7}$ The University of Texas at Austin, 2515 Speedway, Stop C1400, Austin, TX 78712, USA \\ ${ }^{8}$ INAF Osservatorio Astronomico di Roma, Via Frascati 33, I-00040 Monteporzio (RM), Italy \\ ${ }^{9}$ School of Physics \& Astronomy, University of Nottingham, Nottingham NG7 2RD, UK \\ ${ }^{10}$ Centre for Astronomy and Astrophysics, University of Lisbon, P-1349-018 Lisbon, Portugal \\ ${ }^{11}$ Department of Astronomy, University of Massachusetts, Amherst, MA 01003, USA \\ ${ }^{12}$ Max-Planck-Institut für extraterrestrische Physik, Giessen-bachstrasse D-85748 Garching, Germany \\ ${ }^{13}$ Department of Physics and Astronomy, Colby College, Waterville, ME, USA \\ ${ }^{14}$ Institute of Cosmology and Gravitation, University of Portsmouth, Dennis Sciama Building, Burnaby Road, Portsmouth PO1 3FX, UK \\ ${ }^{15}$ NOAO, 950 N. Cherry Avenue, Tucson, AZ 85719, USA \\ Received 2014 October 17; accepted 2015 March 1; published 2015 May 8
}

\begin{abstract}
We combine Hubble Space Telescope (HST)/Wide Field Camera3 (WFC3) imaging and G141 grism observations from the Cosmic Assembly Near-infrared Deep Extragalactic Legacy Survey (CANDELS) and 3D-HST surveys to produce a catalog of grism spectroscopic redshifts for galaxies in the CANDELS/GOODS-South field. The WFC3/ G141 grism spectra cover a wavelength range of $1.1 \leqslant \lambda \leqslant 1.7 \mu \mathrm{m}$ with a resolving power of $R \sim 130$ for point sources, thus providing rest-frame optical spectra for galaxies out to $z \sim 3.5$. The catalog is selected in the $H$-band (F160W) and includes both galaxies with and without previously published spectroscopic redshifts. Grism spectra are extracted for all $H$-band detected galaxies with $\mathrm{H} \leqslant 24$ and a CANDELS photometric redshift $z_{\text {phot }} \geqslant 0.6$. The resulting spectra are visually inspected to identify emission lines, and redshifts are determined using crosscorrelation with empirical spectral templates. To establish the accuracy of our redshifts, we compare our results against high-quality spectroscopic redshifts from the literature. Using a sample of 411 control galaxies, this analysis yields a precision of $\sigma_{\mathrm{NMAD}}=0.0028$ for the grism-derived redshifts, which is consistent with the accuracy reported by the 3D-HST team. Our final catalog covers an area of $153 \mathrm{arcmin}^{2}$ and contains 1019 redshifts for galaxies in GOODS-S. Roughly $60 \%$ (608/1019) of these redshifts are for galaxies with no previously published spectroscopic redshift. These new redshifts span a range of $0.677 \leqslant z \leqslant 3.456$ and have a median redshift of $z=1.282$. The catalog contains a total of 234 new redshifts for galaxies at $z>1.5$. In addition, we present 20 galaxy pair candidates identified for the first time using the grism redshifts in our catalog, including four new galaxy pairs at $z \sim 2$, nearly doubling the number of such pairs previously identified.
\end{abstract}

Key words: catalogs - galaxies: high-redshift - techniques: spectroscopic

Supporting material: machine-readable and VO table

\section{INTRODUCTION}

Accurate galaxy redshifts are vital to studying how the physical properties and environments of galaxies evolve over cosmic time. While high resolution, ground-based spectroscopy has traditionally provided the most reliable redshifts, these observations are time consuming for faint sources and are subject to the limited wavelength sensitivity of optical spectrographs, making it difficult to extend large redshift surveys beyond $z \sim 1.2$ (Davis et al. 2007; Lilly et al. 2007). Near-infrared (NIR) spectrographs are now pushing the redshift frontier into the so-called redshift desert (Trump et al. 2013; Sanders et al. 2014; Silverman et al. 2014; Wisnioski et al. 2014), however at these wavelengths

\footnotetext{
${ }^{16}$ Hubble Fellow.
}

ground-based observations are subject to contamination from atmospheric $\mathrm{OH}$ lines. Photometric redshift estimates, on the other hand, can provide redshifts for large samples of galaxies, including relatively faint systems, at a lower observational cost compared to spectroscopy. However, even the best photometric redshifts have errors of a few percent and are subject to catastrophic outliers for rare sources, such as active galactic nuclei, if their unique spectral energy distributions (SEDs) and/ or strong emission lines are not properly modeled a priori (Salvato et al. 2009, 2011).

Slitless grism spectroscopy with the Wide Field Camera 3 (WFC3) on board the Hubble Space Telescope (HST) now provides a powerful alternative to ground-based spectroscopy and SED modeling for measuring distant redshifts. The slitless nature of the WFC3/IR grism offers the ability to obtain a spectrum of each galaxy in the detector's field of view, while 


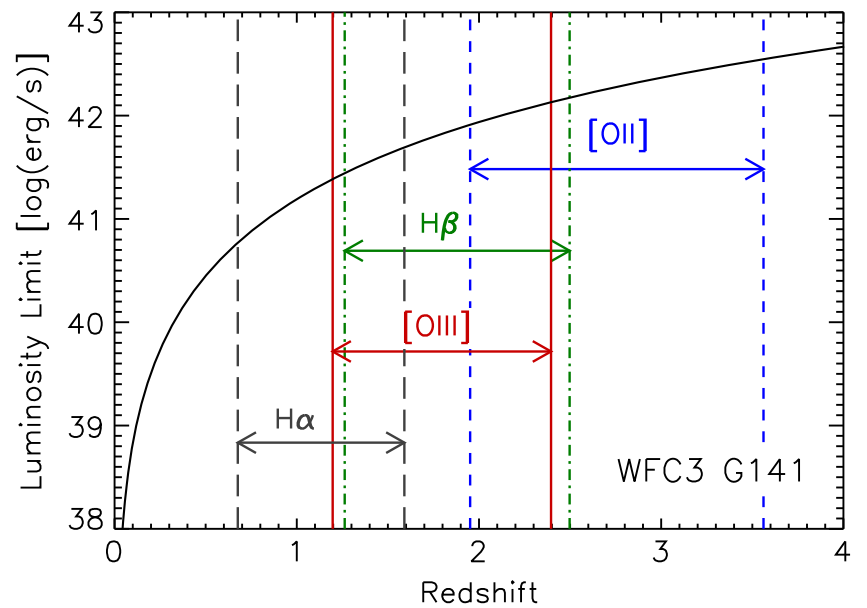

Figure 1. Line luminosity limits for detecting the $\mathrm{H} \alpha, \mathrm{H} \beta,\left[\mathrm{O}_{\mathrm{III}}\right]$, and $\left[\mathrm{O}_{\mathrm{II}}\right]$ emission lines with two orbits of the HST/WFC3 G141 grism (corresponding to a line flux limit of $3 \times 10^{-17} \mathrm{erg} \mathrm{s} \mathrm{cm}^{-2}$ ). Dashed vertical lines indicate the redshift ranges at which various lines are visible in the G141 sensitivity window $(1.1-1.7 \mu \mathrm{m})$.

the significantly reduced background levels compared to the ground means emission lines can be detected for relatively faint galaxies with modest exposure times (e.g., Atek et al. 2011; Trump et al. 2011; Straughn et al. 2011; van der Wel et al. 2011; Brammer et al. 2012). In addition, the NIR sensitivity of WFC3 provides access to many important rest-frame optical emission lines over a wide range of redshifts, from $H \alpha$ down to $z=0.7$ to $[\mathrm{O}$ II $] \lambda 3727$ at $z=3.4$. Figure 1 shows the detectability of emission lines with two-orbit depth G141 grism observations at various redshift ranges. Despite the low spectral resolution $(R \sim 130)$ of the WFC3 grism, the resulting redshift accuracy is an order of magnitude better than typical photometric redshift errors (Brammer et al. 2012).

In this paper, we combine imaging and photometric redshifts from the Cosmic Assembly Near-infrared Deep Extragalactic Legacy Survey (CANDELS; Grogin et al. 2011; Koekemoer et al. 2011) and WFC3/IR grism observations from the 3DHST survey (Brammer et al. 2012) to produce a new grism spectroscopic redshift catalog for $H$-band selected galaxies in the GOODS-South field. The catalog contains emission-line redshifts for 608 sources which have no previously published spectroscopic redshifts and contains 234 new redshifts at $z>1.5$. The paper is organized as follows: in Section 2 we introduce the data sets used in constructing the redshift catalog. In Section 3 we present the methodology used to inspect the grism spectra and measure redshifts. In Section 4 we present an overview of the redshift catalog and its key properties, provide an analysis of the accuracy of the redshift measurements, and use the new redshifts to identify close galaxy pair candidates. Finally, in Section 5 we summarize our work. Throughout this paper, we adopt the Chabrier IMF and the following cosmology: $H_{0}=70 \mathrm{~km} \mathrm{~s}^{-1} \mathrm{Mpc}^{-1}, \Omega_{M}=0.3, \Omega_{\Lambda}=0.7$. All magnitudes are in the AB system.

\section{OBSERVATIONS AND SAMPLE SELECTION}

\subsection{Optical and Infrared Imaging}

Our parent sample is drawn from the $H$-band selected photometric catalog of Guo et al. (2013), which made use of HST/WFC3 imaging of the GOODS-S field from three

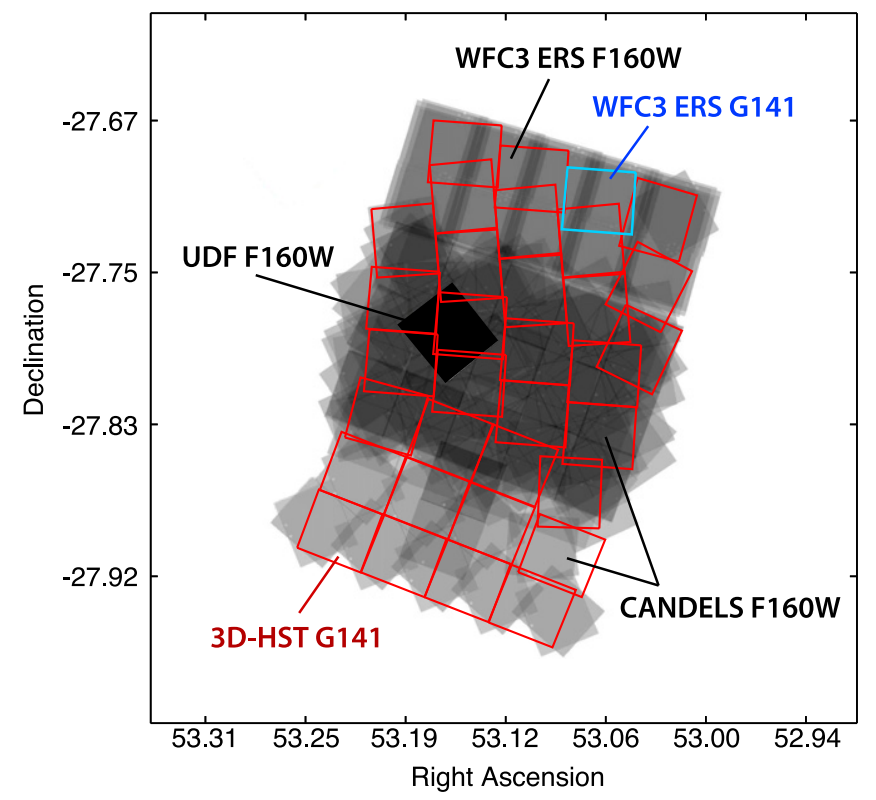

Figure 2. Layout in the GOODS-S field of the WFC3 F160W imaging and G141 grism observations used in this study. The imaging comes from the CANDELS, WFC3 ERS, and HUDF09 programs, while the grism observations come from the 3D-HST (red) and WFC3 ERS programs (blue).

programs: CANDELS, the WFC3 Early Release Science program (ERS; Windhorst et al. 2011), and the HUDF09 program (Bouwens et al. 2010). The location of the WFC3 imaging from these three programs is shown in Figure 2. CANDELS has observed GOODS-S using a two-tiered Wide +Deep strategy. The Deep region covers the central third of the GOODS-S area (55 $\operatorname{arcmin}^{2}$; Giavalisco et al. 2004) with 3, 4, and 6 orbits of F105W, F125W, and F160W imaging, respectively. The Wide region covers the southern third of the field with two-orbit depth imaging in all three bands. The ERS program covers the northern third of GOODS-S with twoorbit depth imaging in the F098M, F125W, and F160W bands. Finally, an area of $4.6 \mathrm{arcmin}^{2}$ in GOODS-S, the Hubble Ultra Deep Field, is covered by very deep 24, 34, and 53 orbits of F105W, F125W, and F160W imaging. The CANDELS team carried out a consistent reduction of the WFC3 imaging from all of these programs; for details we refer readers to Koekemoer et al. (2011).

The GOODS-S field has also been observed in the optical with the Advanced Camera for Surveys on HST as part of the GOODS Hubble Treasury Program (P.I. M. Giavalisco) in the $B, v, i$, and $z$ bands with a total exposure time of 7200, 5450, 7028 , and 18,232 s. For this study, we made use of the publicly available, version v3.0 mosaicked images from the GOODS Treasury Program. In the mid-infrared, we make use of Spitzer/ IRAC 3.6 and $4.5 \mu \mathrm{m}$ imaging from the Spitzer Extended Deep Survey (SEDS; P.I. G. Fazio; Ashby et al. 2013), which reaches total $3 \sigma$ depths of $\sim 26 \mathrm{AB}$ mag.

In addition to the observations described above, the GOODS-S region has been targeted for some of the deepest ground-based observations ever taken, ranging from the $U$ band (Nonino et al. 2009) to the $K$ band (Fontana et al. 2014). A detailed description of these data sets can be found in Grogin et al. (2011) and Guo et al. (2013). 


\subsection{Photometry and Photometric Redshifts}

For this study, we make use of the CANDELS photometric catalog of Guo et al. (2013). The catalog is $H$-band selected using a "max-depth" image that combines all available F160W in the GOODS-S field. The catalog contains 34,930 unique sources and is $50 \%$ complete at $H \sim 26$. Multiwavelength photometry is obtained for the available HST bands using a modified version of SExtractor (Bertin \& Arnouts 1996; see Galametz et al. 2013) using the F160W observations as the detection image. For Spitzer/IRAC imaging and ground-based observations of mixed resolution, the TFIT software (Laidler et al. 2007) was employed to obtain PSF-matched photometry. Further details on the CANDELS multiwavelength photometry catalogs can be found in Guo et al. (2013) and Galametz et al. (2013).

Photometric redshifts for each source were generated from SED modeling using the photometry catalog of Guo et al. (2013). A hierarchical Bayesian approach was employed in which the full photometric redshift probability distribution from 11 independent CANDELS investigators are combined to produce a more accurate redshift estimate. The detailed description of this process can be found in Dahlen et al. (2013). The photometry used ranged from the $U$-band to the Spitzer/IRAC $4.5 \mu \mathrm{m}$ filter; see Guo et al. (2013). The resulting photometric redshifts are found to be accurate to the $2.9 \%$ level and have an outlier fraction (OLF) of $9.1 \%$ when compared with a sample of available spectroscopic redshifts.

\subsection{WFC3/IR Grism Data}

GOODS-S contains near complete spectroscopic coverage in the NIR with two-orbit depth HST/WFC3 G141 grism observations taken by the 3D-HST survey (PI: P. van Dokkum; Brammer et al. 2012) and the WFC3 ERS program (Straughn et al. 2011), corresponding to a limiting line flux of $3 \times 10^{-17} \mathrm{erg} \mathrm{s} \mathrm{cm}^{-2}$. The locations of the G141 observations in the GOODS-S field are shown in Figure 2. The publicly available data were reduced using the aXe software package (Kümmel et al. 2009) to produce two- and one-dimensional wavelength- and flux-calibrated spectra. Spectra were reduced using the default (V2.0) aXe parameters. This means we use a single sky background and do not account for the background fluctuations which typically affect the WFC3 grisms (Brammer et al. 2014). (The GOODS-S G141 observations have lower overall background than the other CANDELS / 3D-HST fields, although the GOODS-S background can vary from $1-2 \mathrm{e}^{-} / \mathrm{s}$ within a single pointing, see Appendix B of Brammer et al. 2012.) The extraction window was set to be four times the object size projected perpendicular to the dispersion direction, where object size is measured from the F140W image using SExtractor. The spectra each cover a wavelength range of $1.1 \leqslant \lambda \leqslant 1.7 \mu \mathrm{m}$ with a resolving power $R \sim 130$ (46.5 ̊/pixel) for point sources. For each observation with the grism, an accompanying direct F140W image is taken to determine the wavelength zero-point for each spectrum. The uncertainty in the zero-point and the dispersion are $8 \AA$ and $0.06 \AA$ /pixel respectively. The dispersion correlates to $\sim 1000 \mathrm{~km} \mathrm{~s}^{-1}$ for $\mathrm{H} \alpha$ at $z>1$. The total exposure time for each F140W direct image is $812 \mathrm{~s}$ and the total exposure time for each G141 grism image ranges between 4511 and $5111 \mathrm{~s}$.

Finally, we registered the grism observations to the CANDELS imaging in the field by running SExtractor on the
F140W direct images and cross-matched the resulting source catalog to the CANDELS F160W catalog of Guo et al. (2013). Through this cross-matching we derived an astrometric correction for each individual 3D-HST tile; the average derived correction was 0.163 in $\Delta \alpha$ and 0.248 in $\Delta \delta$.

\subsection{Sample Selection}

Our initial sample consists of 4511 sources selected from the catalog of Guo et al. (2013) based on the following criteria:

1. $H$-band magnitude: $H_{\mathrm{F} 160 \mathrm{w}} \leqslant 24$

2. Photometric redshift: $z_{\text {phot }} \geqslant 0.6$.

These criteria are chosen such that prominent emission features fall within the sensitivity window of the G141 grism, but also such that the number of sources to be inspected does not become unmanageably large (e.g., increasing the magnitude cut to $H_{\mathrm{F} 160 \mathrm{w}} \leqslant 25$ more than doubles the sample size.) It is worth noting that as a result of these selection criteria, sources that are continuum-faint but have high-equivalent width emission lines may be missed from our initial selection. The same is true for sources with catastrophic failures in their photometric redshift estimates.

To determine which sources in our initial sample have preexisting spectroscopic redshifts, we compared the sample against a recent compilation of published spectroscopic redshifts in the GOODS-S field (N. Hathi 2015, private communication). This compilation contains redshifts from various sources including Croom et al. (2001), Daddi et al. (2004), Le Fèvre et al. (2004), Strolger et al. (2004), Szokoly et al. (2004), van der Wel et al. (2004), Wolf et al. (2004), Mignoli et al. (2005), Kriek et al. (2007), Ravikumar et al. (2007), Vanzella et al. (2008), Huang et al. (2009), Vanzella et al. (2009), Wuyts et al. (2009), Balestra et al. (2010), Silverman et al. (2010), Xue et al. (2011), Cooper et al. (2012), Kurk et al. (2012), Trump et al. (2013), and the ESO GOODS/CDF-S Master Catalog. ${ }^{17}$ We refer to this compilation as the Master Spectroscopic Catalog hereafter. Based on this comparison, we define two samples: a primary sample consisting of 3007 sources which do not appear in the master spectroscopic catalog and a secondary sample of 1504 sources which have published spectroscopic redshifts. In the following sections, we analyze the grism spectra of both samples in an identical manner (i.e., with no prior knowledge of any published spectroscopic redshift) and use the secondary sample to test the accuracy of our grism-derived redshifts (see Section 4.2).

Of the 4511 sources in the initial sample, 2314 sources in the primary sample and 1226 sources in the secondary sample fall within the 3D-HST G141 footprint and are detected in the F140W imaging. For the sources in the primary sample, we extracted 2723 unique grism spectra from the 36 individual 3DHST and ERS pointings, with 343 sources being identified in multiple pointings. We extracted 1464 unique spectra for the secondary sample, with 224 sources being identified in multiple pointings.

\section{REDSHIFT MEASUREMENT}

The extracted 2D and 1D spectra for each object in our samples were visually inspected using the SpecPro IDL

\footnotetext{
17 Available online at http://www.eso.org/sci/activities/garching/projects/ goods/MasterSpectroscopy.html.
} 
software package Masters \& Capak (2011). In addition to the spectrum of each source, SpecPro also provides the user with the ability to display thumbnail images of the source being inspected, a plot of available photometry with an accompanying SED fit, and the location and width of the extraction window. To aid in the identification of emission lines, SpecPro provides the predicted location of various emission and absorption features based on the input photometric redshift for the source. Source spectra were also inspected for contamination from overlapping spectra using an estimate supplied by aXe which is indicated atop the 1D spectrum. It should be noted that no attempt was made to model or subtract contamination from each spectrum, although emission lines from neighboring sources were identified using the photometric or spectroscopic redshift of the contaminating source and contamination from zeroth order spectra is indicated by the contamination estimate supplied by aXe. Any source which exhibited excessive contamination or had data quality issues (e.g., significantly incomplete spectrum due to the spectrum being dispersed off the edge of the detector or spectra which overlap with defective portions of the detector) was removed from the sample. These sources accounted for roughly $17 \%$ of all 4187 extracted spectra in the primary and secondary samples.

During inspection, any visible spectral features were roughly fit manually and subsequently fit via cross-correlation with spectral templates provided in the software to determine the redshift of the source. SpecPro's cross-correlation method is adapted from the cross-correlation routines originally written for the SDSS spectral reduction package, which follows the technique of Tonry \& Davis (1979). When the automated cross-correlation failed, the redshift was determined manually by fitting the peaks of the template emission features to those observed in the grism spectrum. This was done for less than $5 \%$ of sources. The templates used for cross-correlation are taken from the VVDS (Le Fèvre et al. 2005) and PEGASE (Fioc \& Rocca-Volmerange 1997) templates. The emission lines most often used for identification were $\mathrm{H} \alpha /[\mathrm{N}$ II $] \lambda \lambda 6550+6584$ and $\left[\mathrm{S}_{\mathrm{II}}\right] \lambda \lambda 6717+6731, \mathrm{H} \beta$ and $[\mathrm{O} \mathrm{III}] \lambda \lambda 4959+5007$, and $[\mathrm{O} \mathrm{II}]$ $\lambda 3727$. The spectral resolution of the grism is such that the above sets and duplexes are not resolved, but it is enough to produce certain distinguishing profiles that aid in differentiating each from other strong lines (e.g., the asymmetric profile of [O III $] \lambda \lambda 4959+5007$.)

Upon inspection, the derived redshift of each source was assigned a quality flag based on the strength and number of the identified emission lines and the agreement with existing photometric redshift estimates. If a source was identified in multiple pointings and therefore assigned multiple redshifts, the higher quality redshift was always chosen. In the case where multiple redshifts of the same quality exist, the redshift of the source was taken to be the average of the individual redshifts. The quality scheme for the derived redshifts is as follows.

1. 4.0: Multiple high $\mathrm{S} / \mathrm{N}$ emission lines.

2. 3.0: Combination of high and low $\mathrm{S} / \mathrm{N}$ emission lines.

3. 2.5: Single high $\mathrm{S} / \mathrm{N}$ emission line and redshift agrees with $68 \%$ confidence interval of photometric redshift.

4. 2.0: Single high $\mathrm{S} / \mathrm{N}$ emission line and redshift does not agree with $68 \%$ confidence interval of photometric redshift.

Examples of spectra correlating to each quality flag can be seen in Figure 3. While quality 3.0 and 4.0 redshifts are the most reliable, given the multiple emission lines identified, we show in Section 4.2 that sources assigned a quality of 2.0 or 2.5 demonstrate excellent agreement with prior spectroscopic redshift measurements.

\section{RESULTS}

\subsection{Catalog Properties}

Upon inspection and classification of the 2411 grism spectra in the primary sample, we have identified 608 sources with visible emission lines for which redshifts could be measured. Of these, 45 exhibited multiple high $\mathrm{S} / \mathrm{N}$ emission lines (quality 4.0), 181 exhibited multiple emission lines with some low S/N emission lines (quality 3.0), 293 exhibited a single high $\mathrm{S} / \mathrm{N}$ emission line whose redshift agrees with the $68 \%$ confidence interval of the CANDELS photometric redshift estimate (quality 2.5), and 89 exhibited a single high $\mathrm{S} / \mathrm{N}$ emission line which does not agree with the $68 \%$ confidence interval of the photometric redshift (quality 2.0). In the secondary sample, we have identified 411 sources with visible emission lines for which redshifts could be measured. Of these, 35 exhibited multiple high $\mathrm{S} / \mathrm{N}$ emission lines (quality 4.0), 167 exhibited multiple emission lines with some low S/N emission lines (quality 3.0), 157 exhibited a single high $\mathrm{S} / \mathrm{N}$ emission line whose redshift agrees with the $68 \%$ confidence interval of the CANDELS photometric redshift estimate (quality 2.5), and 52 exhibited a single high $\mathrm{S} / \mathrm{N}$ emission line which does not agree with the $68 \%$ confidence interval of the photometric redshift (quality 2.0).

The final catalog contains 1019 grism redshifts for galaxies brighter than $H=24$ in the GOODS-S field. Roughly $60 \%$ $(608 / 1019)$ of the redshifts are new, in that these galaxies have no previously published spectroscopic redshift. The new redshifts span a range of $0.677 \leqslant z \leqslant 3.456$ and have a median redshift of $z=1.282$. The catalog contains a total of 234 new redshifts for galaxies at $z \geqslant 1.5$. The spatial distribution of the 608 galaxies with new redshifts can be seen in Figure 4 and their redshift distribution is shown in Figure 5. In addition, the stellar mass distribution of these 608 galaxies is shown in Figure 6. Here masses are calculated by SED modeling using photometry from the Guo et al. (2013) catalog as described in B. Mobasher et al. (2014, in preparation). We find that galaxies with new grism redshifts in our catalog are, on average, three times less massive than their counterparts with literature redshifts at $z \sim 1-2$.

Over the magnitude range of our primary sample $(H<24)$, we find that our ability to successfully measure a redshift is not strongly dependent on the $H$-band magnitude of the source. Figure 7 shows the magnitude distribution of sources in our primary sample along with the redshift success rate in each magnitude bin (defined as the ratio of the number of sources with grism redshifts of quality $\geqslant 2.0$ to the number of all sources in our initial sample in a given bin). Over the magnitude range $22<H<24$, our success rate ranges from 20 to $30 \%$, showing only a mild decrease for our faintest sources. Also shown in Figure 7 is our success rate as a function of redshift. Here we find a steady decrease from 35 to $10 \%$ in the redshift range $2.0<z<2.75$. This is likely due to [O III] and $\mathrm{H} \beta$ shifting beyond $1.7 \mu \mathrm{m}$ at $z=2.4$ and 2.5, 

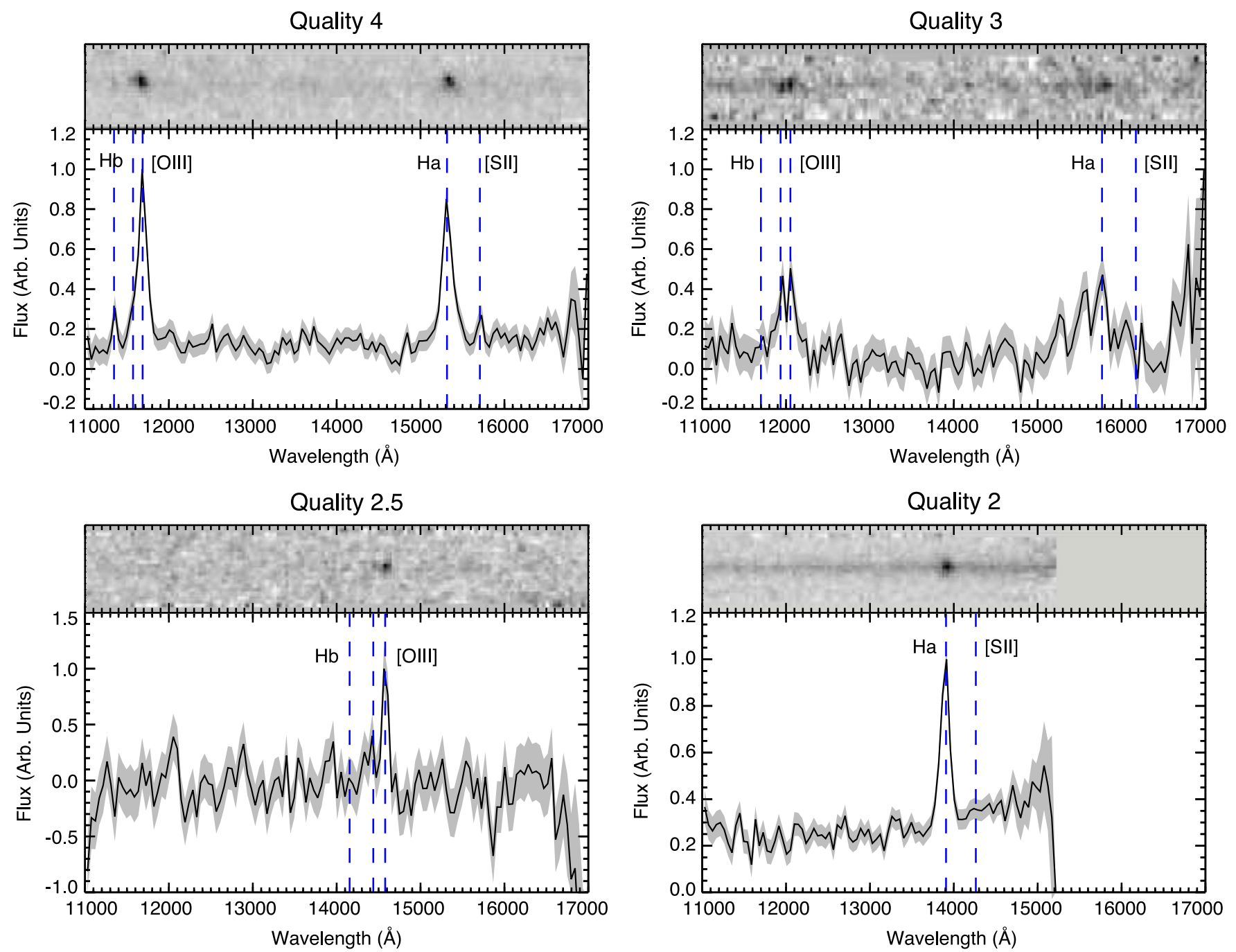

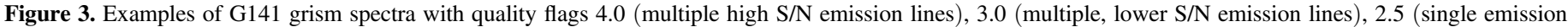

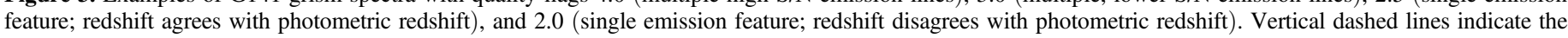
location of major emission features.

respectively, leaving $[\mathrm{O}$ II] as the single emission line visible in the G141 sensitivity window at $z>2.5$.

We have investigated the nature of the sources for which a grism redshift could not be determined due to the lack of visible emission lines and find them to be a combination of bright, quiescent galaxies and faint star-forming systems. In Figure 8, we show the location of these galaxies on a UVJ diagram (Williams et al. 2009). This phase-space separates blue, star-forming galaxies from redder systems that are heavily dust extinguished or passively evolving. For this analysis, rest-frame colors were determined with the EAZY code (Brammer et al. 2008) using the observed photometry from Guo et al. (2013) and the CANDELS photometric redshift catalog. Of the galaxies which lack visible emission lines, we find that $33.0 \%$ are dusty or quiescent $\left(U-V_{\text {rest }}>1.3\right)$. This is nearly three times greater than the $12.4 \%$ of galaxies with measured grism redshifts that have similar rest-frame colors. The remaining $67.0 \%$ are blue, starforming systems $\left(U-V_{\text {rest }}<1.3\right)$ that are predominately faint $(64.5 \%$ are fainter than $H \sim 23$; the same is true for only $23.7 \%$ of the passive/dusty population). We therefore conclude that objects which failed to yield a grism redshift are largely a combination of quiescent galaxies that lack emission lines and star-forming galaxies with emission lines fainter than the detection limit of the grism observations.

\subsection{Redshift Accuracy}

To evaluate the accuracy of our measured redshifts, we first examine the agreement of the new grism redshifts with the CANDELS photometric redshifts. This is shown in the left panel of Figure 9. We to quantify the accuracy of the catalog via the parameter $\sigma_{\mathrm{NMAD}}$, defined as $1.48 \times$ median $\left(|\Delta z| /\left(1+z_{\text {phot }}\right)\right)$. The grism redshifts show good agreement with the photometric redshifts, with $\sigma_{\mathrm{NMAD}}=0.0236$ and an $\operatorname{OLF}\left(|\Delta z| /\left(1+z_{\text {phot }}\right) \geqslant 0.15\right)$ of 0.0150 . These values are comparable to the accuracy of the photometric redshifts when compared against ground-based spectroscopic redshifts, so we may reasonably assume that the majority of this scatter is due to the error in the photometric redshifts. We believe many of the outliers in this evaluation are sources with SEDs contaminated by strong emission lines. A comparison to the work of Hsu et al. (2014), which takes into account intermediate-band Subaru photometry (Cardamone et al. 2010) and the 


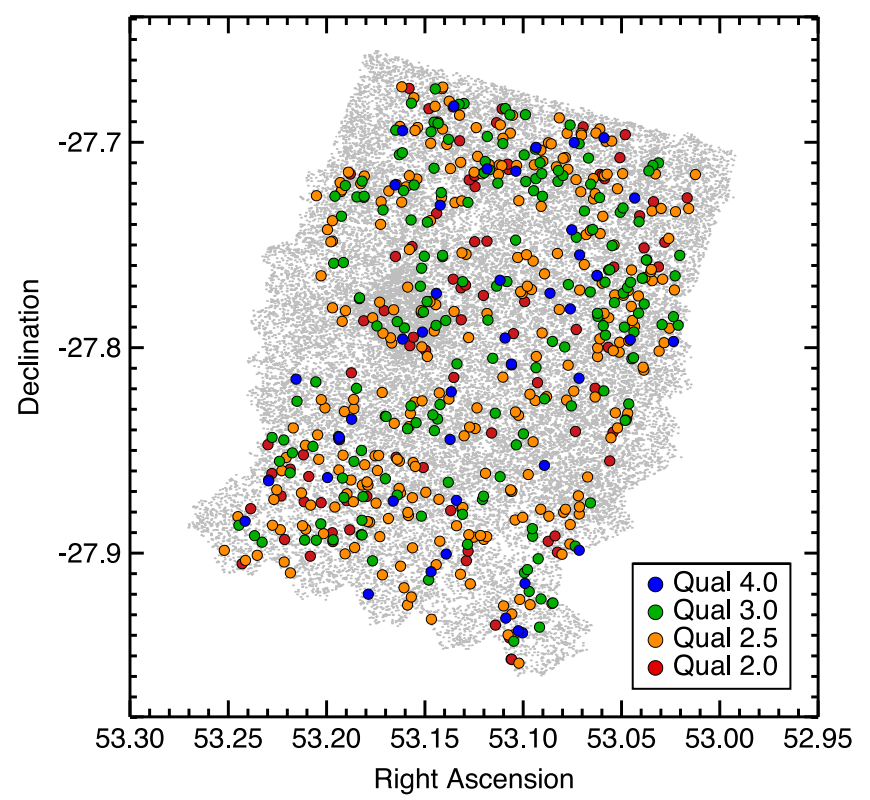

Figure 4. Distribution of sources with new grism redshifts in GOODS-S. Gray points are all sources from the catalog of Guo et al. (2013) and filled circles are sources with grism redshifts, color coded according to their assigned quality; see Section 3 for details.

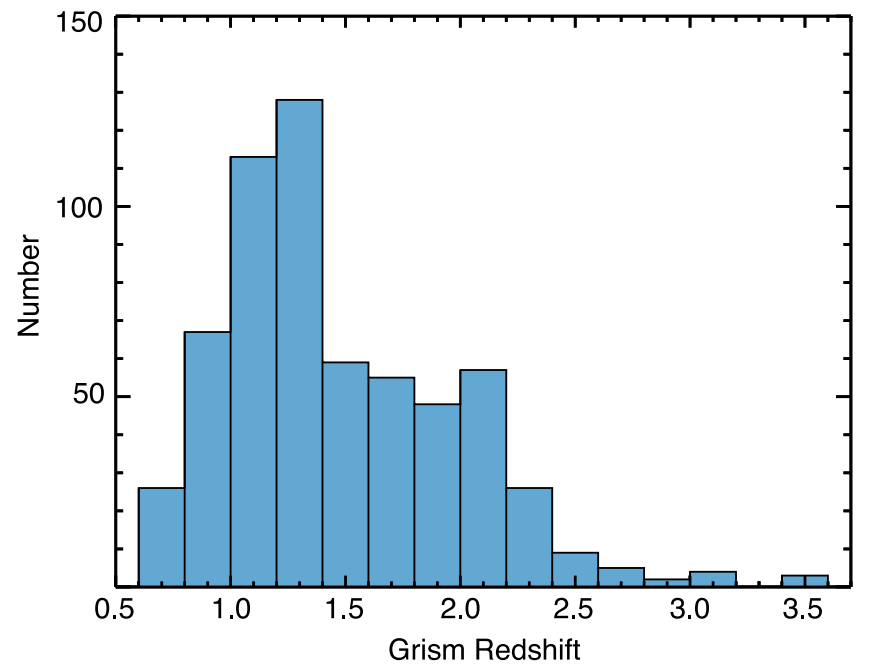

Figure 5. Distribution of the 608 new grism redshifts in GOODS-S. The redshfits span a range of $0.677 \leqslant z \leqslant 3.456$ and have a median redshift of $z=1.282$. There are 234 sources with $z \geqslant 1.5$.

contribution of emission lines in addition to the photometry presented in Guo et al. (2013), reduces the number of outliers in the sample from 9 to just 2 .

To further assess the accuracy of the grism redshifts, we examine the agreement of the secondary sample with the redshifts conained within the master spectroscopic catalog. Each extracted spectrum was inspected using the same methods employed for the primary sample; i.e., we used the photometric redshifts to aid in our redshift determination but had no knowledge of the published spectroscopic redshifts. This process resulted in 411 successful grism redshift determinations. A comparison of the grism and ground-based spectroscopic redshifts is shown on the right panel of Figure 9. This comparison yields a scatter of $\sigma_{\mathrm{NMAD}}=0.0028$ with an OLF of 0.0098. Compared to the work of Brammer et al. (2012), we

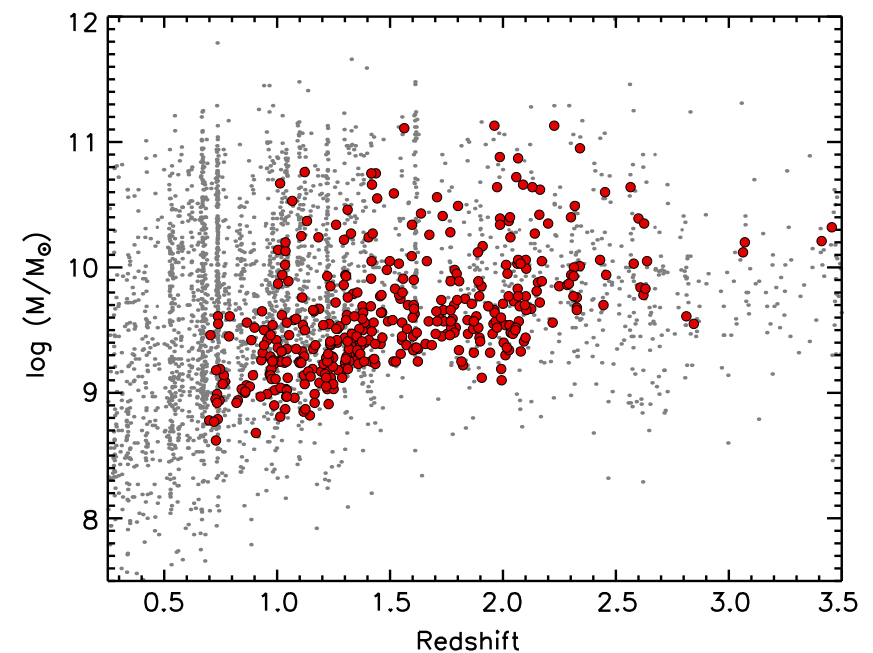

Figure 6. Mass distribution as a function of spectroscopic redshift for galaxies in the GOODS-S field. Galaxies with new grism redshifts from this work are shown as red circles, while galaxies with previously published spectroscopic redshifts are shown as gray points.

find excellent agreement with the accuracy reported by the $3 \mathrm{D}$ $H S T$ team $\left(\sigma_{\mathrm{NMAD}}=0.0035\right)$.

As a further test of the accuracy of our catalog, we have compared our grism redshifts to those obtained by the WFC3 ERS program. Straughn et al. (2011) obtained 48 redshifts via their G102 and G141 observations, of which 10 sources with the highest quality redshifts meet our magnitude and redshift selection criteria and received quality flags of 2.0 or higher in our inspection. We see excellent agreement between our results and those of Straughn et al. (2011), with a comparison in the manner described above giving a result of $\sigma_{\mathrm{NMAD}}=0.0016$.

We find that the scatter between the grism and ground-based spectroscopic redshifts is not significantly increased $\left(\Delta \sigma_{\mathrm{NMAD}} \approx 0.0001\right)$ for those sources whose redshifts were fit manually versus those fit via the built-in cross-correlation routines in SpecPro. We also find that the scatter does not vary significantly with the quality flag, ranging from $\sigma_{\mathrm{NMAD}}=0.0026$ for quality four redshifts to $\sigma_{\mathrm{NMAD}}=0.0033$ for quality two redshifts. We therefore propose quality 2.0 as the minimum reliable quality for this redshift catalog. In addition, we find no correlation between scatter and the effective radius of the sources as defined in van der Wel et al. (2014).

\subsection{Redshift Catalog}

Starting with a sample of 4511 sources, we have obtained a total of 1019 grism redshifts for galaxies brighter than $H=24$ in the GOODS-S field. Of these, 608 are new redshift measurements for galaxies in our primary sample, which do not have previously published spectroscopic redshifts. The coordinates and redshifts of all 1019 galaxies are listed in Table 1. The details of the table columns are given below.

1. Source ID from Guo et al. (2013).

2. Right ascension (J2000).

3. Declination (J2000).

4. H-band magnitude (AB) from Guo et al. (2013).

5. Redshift derived from G141 grism spectrum.

6. Redshift from Master Spectroscopic Catalog. 

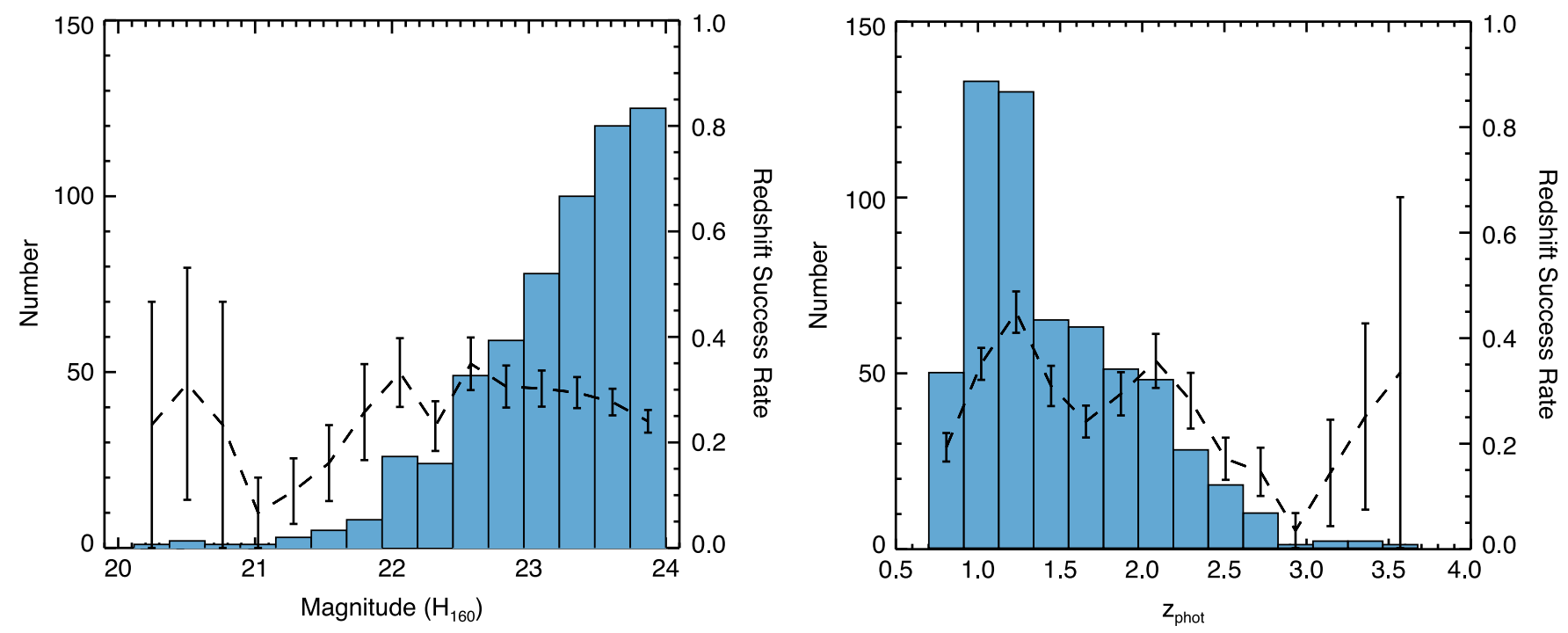

Figure 7. (Left) Magnitude distribution of sources with new grism redshifts. The dashed line represents the redshift "success" rate in each bin with error bars given by number statistics based on the number of sources in that bin. (Right) Redshift distribution of sources with new grism redshifts.

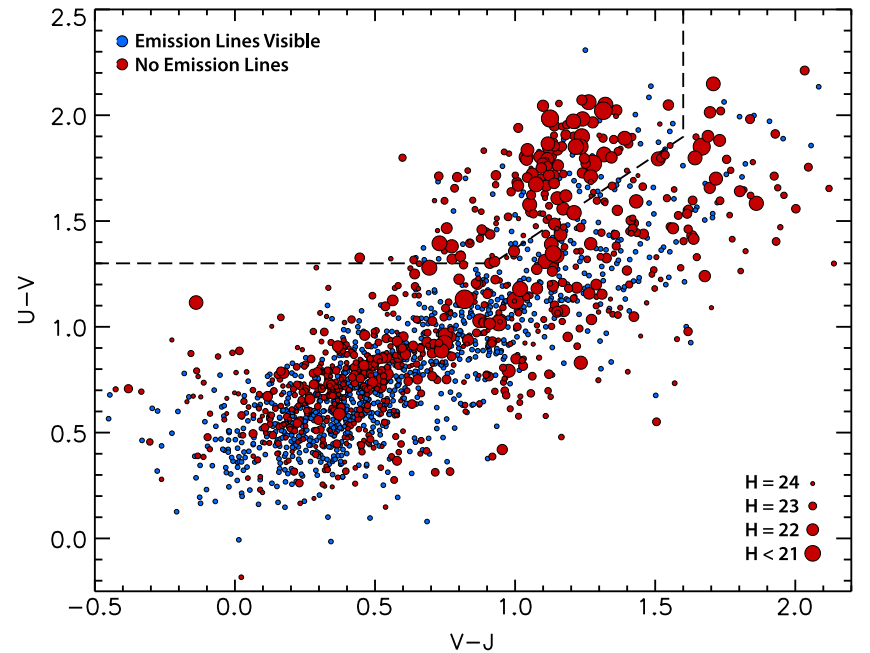

Figure 8. UVJ plot of rest-frame colors for all sources in both the primary and secondary samples. Blue points represent sources for which a grism redshift was successfully obtained and red sources represent sources for which a grism redshift was not obtained with point size scaled according to the source's $H$ band magnitude. The dashed line separates the star-forming "blue cloud" and the quiescent "red sequence." We find that sources without grism redshifts tend to be bright quiescent systems or faint star-forming galaxies.

7 Emission line(s) used for redshift determination.

8. Redshift quality flag (see Section 3 for details).

\subsection{Newly Identified Galaxy Pair Candidates}

In this section, we highlight one of the potential uses for our redshift catalog, namely the identification of close galaxy pairs at $z>1$. Due to the slitless nature of the WFC3 grism, we can now dramatically increase the spectroscopic sampling of starforming galaxies at these redshifts. Even with a spectral resolution of $\delta v \sim 1000 \mathrm{~km} \mathrm{~s}^{-1}$, the redshift accuracy of the G141 grism spectra $\left(\sigma_{\mathrm{z}} /(1+z)=0.3 \%\right)$ is far better than typical photometric redshift errors $(3 \%)$ and the accuracy of low-resolution prism spectroscopy $(1.2 \%)$, which have been used in the past to study the merger rate and environments of galaxies at $z \sim 1$ and beyond, i.e., Patel et al. (2011) and Newman et al. (2012).

Combining the grism redshifts in our catalog with our master spectroscopic catalog, we have identified 20 galaxy pair candidates in GOODS-S with at least one member whose redshift comes from the new WFC3/G141 spectra. To identify galaxy pairs, we inspect the neighbors of each galaxy with a grism redshift of quality 2.0 or greater and define close companions as those that are (1) within a projected distance of $50 \mathrm{kpc}$, and (2) have a redshift difference of $\Delta z /(1+z)<0.03$, or roughly $\delta v \sim 1000 \mathrm{~km} \mathrm{~s}^{-1}$ at $z>1$. Based on these criteria, we find 20 galaxies with potential companions in the CANDELS/ GOODS-S region. Five of the pairs are comprised of two galaxies with new grism redshifts and 15 are grism sources with companions that appear in our master spectroscopic catalog. The sample spans a redshift range of $0.787 \leqslant z \leqslant 2.33$, with four of the pairs identified at $z \sim 2$. This sample represents roughly a factor of 2 increase in the number of such pairs identified with the master spectroscopic catalog alone. On average, the new companions to sources with spectroscopic redshifts are nearly one magnitude fainter in the $H$-band, which highlights the ability of the grism to detect fainter objects than are usually seen via ground-based spectroscopy. In addition, the objects found in these pairs are approximately ten times less massive than objects typically observed in pair studies at this redshift (López-Sanjuan et al. 2013; Tasca et al. 2014) The coordinates and redshifts of the newly detected pair candidates are listed in Table 2.

\section{SUMMARY}

We have constructed a redshift catalog for galaxies in the CANDELS/GOODS-S field using HST/WFC3 G141 grism observations from the 3D-HST survey and WFC3 ERS program. The G141 spectra cover a wavelength range of $1.1 \leqslant \lambda \leqslant 1.7 \mu \mathrm{m}$, which allows for the detection of prominent emission lines over a wide redshift range, from $\mathrm{H \alpha}$ at $z=0.7$ to [O II] $\lambda 3727$ at $z=3.4$. Our catalog is $H$-band selected based on the CANDELS photometry catalog of Guo et al. (2013). Spectra were extracted for all GOODS-S sources which are 

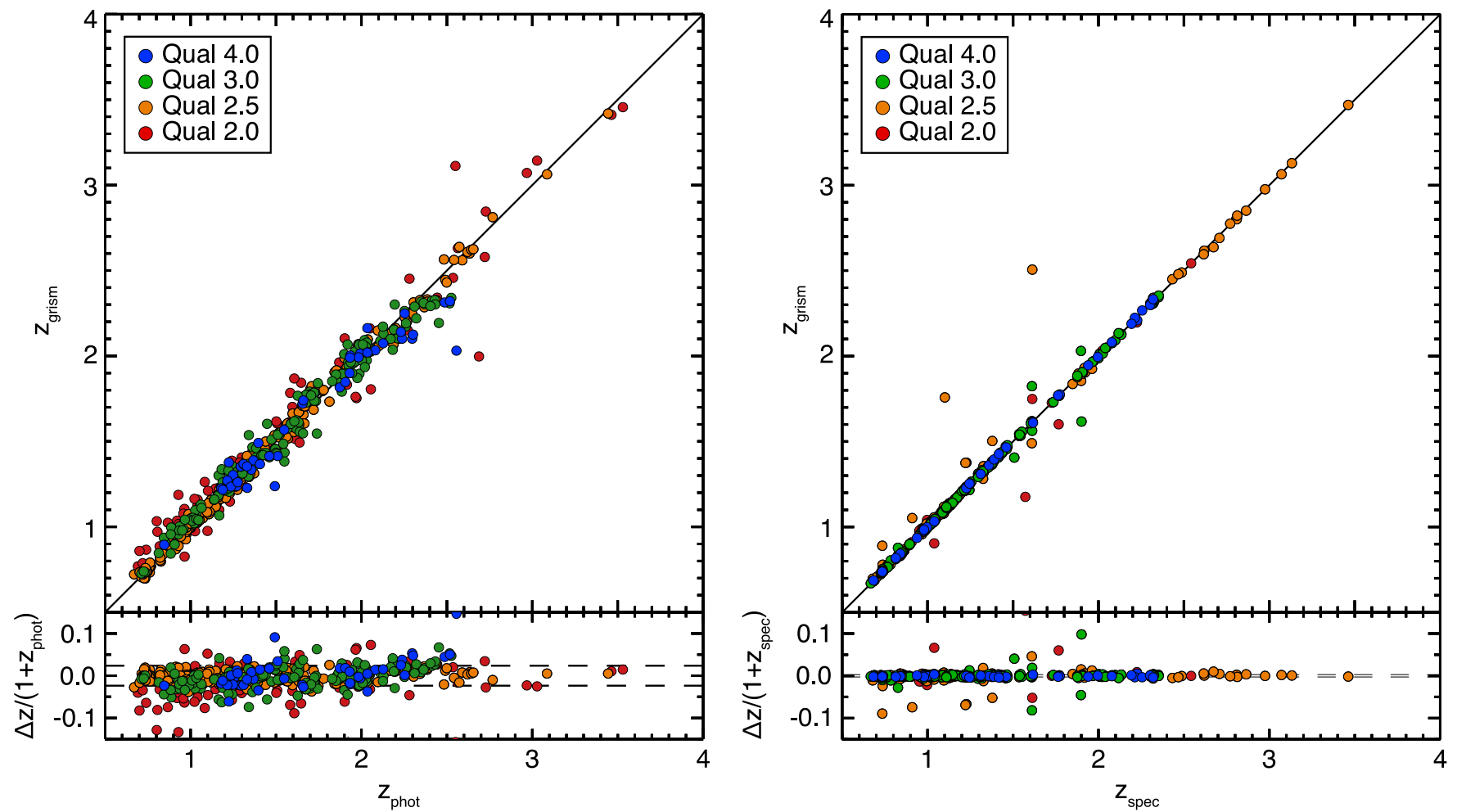

Figure 9. (Left) Grism redshifts from this work vs. CANDELS photometric redshifts. The solid line represents $z_{\text {grism }}=z_{\text {phot }}$, while the dashed lines represent $\sigma_{\mathrm{NMAD}}=0.0236$. (Right) Grism redshifts from this work vs. ground-based spectroscopic redshifts, with $\sigma_{\mathrm{NMAD}}=0.0028$.

Table 1

Grism Redshift Catalog of Sources in GOODS-S

\begin{tabular}{|c|c|c|c|c|c|c|c|}
\hline $\begin{array}{l}\text { Source ID } \\
\text { (1) }\end{array}$ & $\begin{array}{l}\text { R.A. } \\
(2)\end{array}$ & $\begin{array}{c}\text { Decl. } \\
(3)\end{array}$ & $\begin{array}{c}\mathrm{AB}(\mathrm{F} 160 \mathrm{~W}) \\
(4)\end{array}$ & $\begin{array}{l}z_{\text {grism }} \\
(5)\end{array}$ & $\begin{array}{c}z_{\text {spec }} \\
(6)\end{array}$ & $\begin{array}{l}\text { Lines } \\
(7)\end{array}$ & $\begin{array}{l}\text { Qual } \\
(8)\end{array}$ \\
\hline 36 & $03 \quad 32 \quad 24.52$ & $-2757 \quad 12.8$ & 23.54 & 0.733 & $\ldots$ & $\mathrm{H} \alpha$ & 2.5 \\
\hline 54 & $03 \quad 32 \quad 25.47$ & -275705.7 & 23.95 & 1.805 & $\ldots$ & {$[\mathrm{O}$ III] } & 2.0 \\
\hline 55 & $03 \quad 3225.42$ & -275705.9 & 23.74 & 1.753 & $\ldots$ & {$[\mathrm{O}$ III] } & 2.0 \\
\hline 173 & 033225.39 & -275633.9 & 22.97 & 0.683 & 0.684 & $\mathrm{H} \alpha,\left[\mathrm{S}_{\mathrm{III}}\right] \lambda 9532$ & 3.0 \\
\hline 180 & $0332 \quad 25.15$ & -275634.3 & 23.53 & 1.332 & $\ldots$ & $\mathrm{H} \beta,[\mathrm{O}$ III $], \mathrm{H} \alpha$ & 3.0 \\
\hline 218 & 033225.66 & -275628.5 & 22.87 & 1.112 & $\ldots$ & $\mathrm{H} \alpha$ & 2.0 \\
\hline 244 & $0332 \quad 25.18$ & -275623.1 & 23.20 & 1.103 & $\ldots$ & $\mathrm{H} \alpha$ & 2.5 \\
\hline 267 & $03 \quad 32 \quad 25.84$ & -275623.2 & 22.67 & 1.087 & $\ldots$ & $\mathrm{H} \alpha$ & 2.5 \\
\hline 287 & $03 \quad 3224.08$ & -275619.7 & 23.29 & 2.101 & $\ldots$ & $\mathrm{H} \beta,[\mathrm{O} \quad \mathrm{III}]$ & 4.0 \\
\hline 304 & 033224.59 & -275615.9 & 23.20 & 1.267 & $\cdots$ & {$[\mathrm{O}$ III] $], \mathrm{H} \alpha$} & 4.0 \\
\hline 371 & 033222.01 & -275609.6 & 22.62 & 1.777 & $\ldots$ & $\mathrm{H} \beta,[\mathrm{O} \quad \mathrm{III}]$ & 3.0 \\
\hline 394 & $0332 \quad 27.39$ & -275606.0 & 23.81 & 0.826 & $\ldots$ & $\mathrm{H} \alpha$ & 2.0 \\
\hline 472 & 033231.16 & -275557.2 & 23.20 & 0.836 & 0.841 & $\mathrm{H} \alpha$ & 2.5 \\
\hline 482 & $03 \quad 3224.84$ & -275559.9 & 21.96 & 1.052 & 1.049 & $\mathrm{H} \alpha$ & 2.5 \\
\hline 483 & 033235.20 & -275555.8 & 22.93 & 0.997 & $\ldots$ & $\mathrm{H} \alpha$ & 2.5 \\
\hline 489 & 033221.92 & -275555.0 & 23.36 & 3.064 & 3.071 & {$[\mathrm{O}$ II $]$} & 2.5 \\
\hline 494 & 033226.12 & -275553.9 & 23.20 & 2.101 & $\ldots$ & {$[\mathrm{O}$ II] $], \mathrm{H} \beta,[\mathrm{O}$ III] } & 4.0 \\
\hline 513 & 033228.46 & -275554.3 & 22.55 & 1.090 & 1.090 & $\mathrm{H} \alpha$ & 2.5 \\
\hline 528 & $03 \quad 3227.23$ & -275559.8 & 19.86 & 1.096 & 1.089 & $\mathrm{H} \alpha$ & 2.0 \\
\hline 540 & 033226.45 & -275549.7 & 23.11 & 2.093 & 2.099 & {$[\mathrm{O}$ II], [O III] } & 3.0 \\
\hline
\end{tabular}

Note. $\left[\mathrm{O}_{\mathrm{II}}\right]$ and $\left[\mathrm{O}_{\mathrm{III}}\right]$ in column 7 above refer always to the $\left[\mathrm{O}_{\mathrm{II}}\right] \lambda \lambda 3727+3729$ and $[\mathrm{O} \mathrm{III}] \lambda \lambda 4959+5007$ emission line doublets.

(This table is available in its entirety in machine-readable and Virtual Observatory (VO) forms.)

brighter than $H=24$ and have a photometric redshift $z_{\text {phot }} \geqslant 0.6$. Each spectrum was visually inspected, emission lines were identified with the aid of CANDELS photometric redshifts, and redshifts were measured via cross-correlation with empirical spectral templates. Derived redshifts were assigned a quality ranging from 4.0 for sources with multiple strong emission lines, to 2.0 for sources with a single visible emission line. The resulting catalog contains new grism redshifts for 608 galaxies which have no previously published spectroscopic redshift in the GOODS-S field. These redshifts span a range of $0.677 \leqslant z \leqslant 3.456$ and include 234 new redshifts for galaxies at $z \geqslant 1.5$. The catalog also contains 
Table 2

Grism-identified Pairs in GOODS-S

\begin{tabular}{|c|c|c|c|c|c|c|c|c|c|c|}
\hline $\begin{array}{l}\text { Source ID } 1 \\
\text { (Guo et } \\
\text { al. 2013) }\end{array}$ & $\begin{array}{l}\text { Source ID } 2 \\
\text { (Guo et } \\
\text { al. 2013) }\end{array}$ & Source 1 & Source 1 & Source 2 & Source 2 & $z_{1}$ & $z_{2}$ & Angular Sep. & $\begin{array}{c}\text { Proj. } \\
\text { Separation } \\
(\mathrm{kpc})\end{array}$ & $\begin{array}{l}\text { Mass } \\
\text { Ratio }\end{array}$ \\
\hline 722 & 726 & 53.096496 & -27.925050 & 53.096385 & -27.925972 & 1.145 & 1.146 & 3.338 & 27.48 & 0.847 \\
\hline 1708 & 1695 & 53.241265 & -27.903526 & 53.241173 & -27.903265 & 1.050 & 1.045 & 0.984 & 7.963 & 1.086 \\
\hline 2524 & 2526 & 53.182411 & -27.890996 & 53.181927 & -27.891138 & 1.458 & 1.465 & 1.623 & 13.72 & 0.974 \\
\hline 3606 & 3689 & 53.078473 & -27.878643 & 53.078256 & -27.878455 & 0.987 & 0.987 & 0.967 & 7.720 & 0.981 \\
\hline 3707 & 3952 & 53.071376 & -27.877464 & 53.072763 & -27.876321 & 1.099 & 1.100 & 6.034 & 49.31 & 0.845 \\
\hline 5948 & 5983 & 53.055988 & -27.855096 & 53.056169 & -27.855367 & 1.227 & 1.226 & 1.133 & 9.426 & 0.948 \\
\hline 6223 & 6195 & 53.173546 & -27.852423 & 53.175354 & -27.852610 & 0.967 & 0.962 & 5.794 & 45.99 & 0.971 \\
\hline 7129 & 7172 & 53.193578 & -27.843512 & 53.193960 & -27.843195 & 2.003 & 2.011 & 1.668 & 13.96 & 1.011 \\
\hline 8472 & 8452 & 53.114217 & -27.831856 & 53.113214 & -27.832735 & 1.295 & 1.293 & 4.495 & 37.64 & 0.951 \\
\hline 10987 & 11071 & 53.093517 & -27.809712 & 53.093552 & -27.809287 & 2.327 & 2.334 & 1.534 & 12.56 & 0.963 \\
\hline 11160 & 11218 & 53.105939 & -27.808401 & 53.106022 & -27.807946 & 2.014 & 2.020 & 1.659 & 13.87 & 1.007 \\
\hline 16113 & 16275 & 53.100546 & -27.773643 & 53.101308 & -27.772737 & 1.014 & 1.016 & 4.066 & 32.68 & 0.952 \\
\hline 18095 & 18109 & 53.037376 & -27.757329 & 53.037104 & -27.757242 & 2.025 & 2.017 & 0.921 & 7.701 & 1.044 \\
\hline 18967 & 18795 & 53.156543 & -27.750777 & 53.157256 & -27.751523 & 1.605 & 1.607 & 3.517 & 29.80 & 0.908 \\
\hline 21681 & 21576 & 53.205173 & -27.726024 & 53.205371 & -27.726181 & 1.169 & 1.163 & 0.847 & 6.994 & 1.091 \\
\hline 21839 & 21897 & 53.141731 & -27.724507 & 53.142011 & -27.724565 & 1.602 & 1.602 & 0.916 & 7.761 & 0.956 \\
\hline 21932 & 22005 & 53.168360 & -27.723737 & 53.168083 & -27.723513 & 1.248 & 1.245 & 1.196 & 9.971 & 0.949 \\
\hline 22795 & 23970 & 53.058673 & -27.716358 & 53.059861 & -27.716288 & 1.023 & 1.022 & 3.794 & 30.54 & 0.979 \\
\hline 23632 & 24274 & 53.117190 & -27.713409 & 53.116405 & -27.712703 & 1.618 & 1.610 & 3.566 & 30.21 & 0.868 \\
\hline 24564 & 24904 & 53.135636 & -27.682839 & 53.136296 & -27.683073 & 0.787 & 0.787 & 2.266 & 16.92 & 0.974 \\
\hline
\end{tabular}

grism redshifts for 411 galaxies which have existing redshifts in the literature.

We find good agreement between our grism-derived redshifts and existing photometric redshifts from CANDELS $\left(\sigma_{\mathrm{NMAD}}=0.0236\right)$. We have also tested the accuracy of our redshifts by extracting and inspecting the spectra of GOODS-S sources with published spectroscopic redshifts. This analysis was done blind, with only the photometric redshift of each source known during the inspection. Here we find excellent agreement between our redshifts and the published values $\left(\sigma_{\mathrm{NMAD}}=0.0028\right)$. This agreement holds even for redshifts measured with only a single emission line (quality 2.5 and 2.0 in the catalog).

Finally, we use our redshift catalog to identify 20 new galaxy pair candidates at $z=1-2$. These were chosen to have a projected separation of $50 \mathrm{kpc}$ and a velocity offset of $\delta v \sim 1000 \mathrm{~km} \mathrm{~s}^{-1}$. Included in this sample are four new pairs identified at $z \sim 2$.

This work is based on observations taken by the CANDELS Multi-Cycle Treasury Program (GO 12177) and the 3D-HST Treasury Program (GO 12328) with the NASA/ESA HST, which is operated by the Association of Universities for Research in Astronomy, Inc., under NASA contract NAS526555 and by Spitzer, which is operated by the Jet Propulsion Laboratory, California Institute of Technology under a contract with NASA. We also acknowledge partial support from NSF 0808133 and $H S T$-AR $12822.03 \mathrm{~A}$.

\section{REFERENCES}

Ashby, M. L. N., Willner, S. P., Fazio, G. G., et al. 2013, ApJ, 769, 80 Atek, H., Siana, B., Scarlata, C., et al. 2011, ApJ, 743, 121

Balestra, I., Mainieri, V., Popesso, P., et al. 2010, A\&A, 512, A12

Bertin, E., \& Arnouts, S. 1996, A\&AS, 117, 393

Bouwens, R. J., Illingworth, G. D., Oesch, P. A., et al. 2010, ApJL, 709, L133
Brammer, G., Pirzkal, N., McCullough, P., \& MacKenty, J. 2014, Timevarying Excess Earth-glow Backgrounds in the WFC3/IR Channel, Instrument Science Report WFC3 2014-03 (Baltiomre, MD: STScI)

Brammer, G. B., van Dokkum, P. G., \& Coppi, P. 2008, ApJ, 686, 1503

Brammer, G. B., van Dokkum, P. G., Franx, M., et al. 2012, ApJS, 200, 13

Cardamone, C. N., van Dokkum, P. G., Urry, C. M., et al. 2010, ApJS, 189,270

Cooper, M. C., Yan, R., Dickinson, M., et al. 2012, MNRAS, 425, 2116

Croom, S. M., Warren, S. J., \& Glazebrook, K. 2001, MNRAS, 328, 150

Daddi, E., Cimatti, A., Renzini, A., et al. 2004, ApJL, 600, L127

Dahlen, T., Mobasher, B., Faber, S. M., et al. 2013, ApJ, 775, 93

Davis, M., Guhathakurta, P., Konidaris, N. P., et al. 2007, ApJL, 660, L1

Fioc, M., \& Rocca-Volmerange, B. 1997, A\&A, 326, 950

Fontana, A., Dunlop, J. S., Paris, D., et al. 2014, arXiv:1409.7082

Galametz, A., Grazian, A., Fontana, A., et al. 2013, ApJS, 206, 10

Giavalisco, M., Ferguson, H. C., Koekemoer, A. M., et al. 2004, ApJL, 600, L93

Grogin, N. A., Kocevski, D. D., Faber, S. M., et al. 2011, ApJS, 197, 35

Guo, Y., Ferguson, H. C., Giavalisco, M., et al. 2013, ApJS, 207, 24

Hsu, L.-T., Salvato, M., Nandra, K., et al. 2014, arXiv:1409.7119

Huang, J.-S., Faber, S. M., Daddi, E., et al. 2009, ApJ, 700, 183

Koekemoer, A. M., Faber, S. M., Ferguson, H. C., et al. 2011, ApJS, 197, 36

Kriek, M., van Dokkum, P. G., Franx, M., et al. 2007, ApJ, 669, 776

Kümmel, M., Walsh, J. R., Pirzkal, N., Kuntschner, H., \& Pasquali, A. 2009, PASP, 121, 59

Kurk, J., Cimatti, A., Daddi, E., et al. 2012, yCat, 354, 99063

Laidler, V. G., Papovich, C., Grogin, N. A., et al. 2007, PASP, 119, 1325

Le Fèvre, O., Vettolani, G., Paltani, S., et al. 2004, A\&A, 428, 1043

Le Fèvre, O., Guzzo, L., Meneux, B., et al. 2005, A\&A, 439, 877

Lilly, S. J., Le Fèvre, O., Renzini, A., et al. 2007, ApJS, 172, 70

López-Sanjuan, C., le Fèvre, O., Tasca, L. A. M., et al. 2013, A\&A, 553, A78

Masters, D., \& Capak, P. 2011, PASP, 123, 638

Mignoli, M., Cimatti, A., Zamorani, G., et al. 2005, A\&A, 437, 883

Newman, A. B., Ellis, R. S., Bundy, K., \& Treu, T. 2012, ApJ, 746, 162

Nonino, M., Dickinson, M., Rosati, P., et al. 2009, ApJS, 183, 244

Patel, S. G., Kelson, D. D., Holden, B. P., Franx, M., \& Illingworth, G. D. 2011, ApJ, 735, 53

Ravikumar, C. D., Puech, M., Flores, H., et al. 2007, A\&A, 465, 1099

Salvato, M., Hasinger, G., Ilbert, O., et al. 2009, ApJ, 690, 1250

Salvato, M., Ilbert, O., Hasinger, G., et al. 2011, ApJ, 742, 61

Sanders, R. L., Shapley, A. E., Kriek, M., et al. 2014, arXiv:1408.2521

Silverman, J. D., Mainieri, V., Salvato, M., et al. 2010, ApJS, 191, 124

Silverman, J. D., Kashino, D., Arimoto, N., et al. 2014, arXiv:1409.0447

Straughn, A. N., Kuntschner, H., Kümmel, M., et al. 2011, AJ, 141, 14 
Strolger, L.-G., Riess, A. G., Dahlen, T., et al. 2004, ApJ, 613, 200

Szokoly, G. P., Bergeron, J., Hasinger, G., et al. 2004, ApJS, 155, 271

Tasca, L. A. M., Le Fèvre, O., López-Sanjuan, C., et al. 2014, A\&A, 565, A10

Tonry, J., \& Davis, M. 1979, AJ, 84, 1511

Trump, J. R., Weiner, B. J., Scarlata, C., et al. 2011, ApJ, 743, 144

Trump, J. R., Konidaris, N. P., Barro, G., et al. 2013, ApJL, 763, L6

van der Wel, A., Franx, M., van Dokkum, P. G., \& Rix, H.-W. 2004, ApJL, 601, L5

van der Wel, A., Straughn, A. N., Rix, H.-W., et al. 2011, ApJ, 742, 111

van der Wel, A., Franx, M., van Dokkum, P. G., et al. 2014, ApJ, 788, 28
Vanzella, E., Cristiani, S., Dickinson, M., et al. 2008, A\&A, 478, 83

Vanzella, E., Giavalisco, M., Dickinson, M., et al. 2009, ApJ, 695, 1163

Williams, R. J., Quadri, R. F., Franx, M., van Dokkum, P., \& Labbé, I. 2009, ApJ, 691, 1879

Windhorst, R. A., Cohen, S. H., Hathi, N. P., et al. 2011, ApJS, 193, 27

Wisnioski, E., Förster Schreiber, N. M., Wuyts, S., et al. 2014, arXiv: 1409.6791

Wolf, C., Meisenheimer, K., Kleinheinrich, M., et al. 2004, A\&A, 421, 913

Wuyts, S., van Dokkum, P. G., Franx, M., et al. 2009, ApJ, 706, 885

Xue, Y. Q., Luo, B., Brandt, W. N., et al. 2011, ApJS, 195, 10 